Methods: We prospectively collected data from DUs of the hands evaluated in our dedicated wound-care outpatient clinic from October 2018 to August 2019. Fifty-five patients were enrolled (50 females, 18 with limited-SSc and 37 with diffuse-SSc, mean age $62.3 \pm 17.2$ years). For every DU we collected: presence/absence of calcinosis, pathogenesis (spontaneous, post-traumatic), area of DU, location (fingertip, periungual area, metacarpophalangeal, proximal/distal interphalangeal-PIP/DIP), VAS-pain at the baseline and after two weeks, local signs of infection (edema, redness), deep wound swab results and time to the healing. Additionally, we calculated the wound-bed score (WBS), at the baseline and we correlated the total score with the time of healing. All the ulcers were managed with weekly treatment following a definite protocol: wound cleansing, disinfection, mechanic debridement, application of antiseptic dressing.

Results: Out of 98 DUs evaluated, 24 (24.5\%) were associated with SC. Patients with SC were older than those without calcinosis $(67.1+-16.9$ vs $59.4+-16.9 \mathrm{p}<0.05)$ and were more frequently affected by Ic-SSc $(18-75 \%$ vs $6-25 \% \mathrm{p}<0.001)$. There were no significant differences between the mean areas of DUs (SC $22 \mathrm{~mm}^{2}$ vs non-calcinosis $30.8 \mathrm{~mm}^{2}$ ) neither in the localization of the ulcers: fingertip (14-61\% vs $34-49.3 \%)$, periungual area $(4-17.4 \%$ vs $16-23.2 \%)$, PIP (2-9\% vs $13-18.9 \%)$, DIP (2-9\% vs $9-13 \%)$ and MCP $(1-4 \%$ vs 4-5.8\%). The VAS-pain was not statistically different at the baseline (6.0 for SC vs 5.4), neither after 2 weeks (3.8 vs 3.2). Although the presence of local signs of infection was similar $(5-20.8 \%$ vs $14-18.9 \%)$, the positivity for the wound swab was higher in SC compared with those without calcinosis $(6-26.1 \%$ vs 9-11.5\%; $p=0.05)$.

All the DUs treated in our outpatient clinic healed but those with SC required more weeks $(10.4 \pm 7.9$ vs. $7.13 \pm 5.7 ; p=0.03)$. The WBS was similar in the two groups (8.96+-0.46 in SC vs $9.43+-0.33$ ) and was negatively correlated with the time of healing $(r=-0.24, p=0.02)$

Conclusion: Although DUs with calcinosis have a different pathogenesis compared to those without SC, the location, dimensions and DU-related pain are similar in both groups. Despite these aspects, DUs associated with calcinosis are more prone to be infected and require more time to heal; the WBS may represent a simple, easy-calculated score to predict the time for DUs healing. The presence of calcinosis may represent a negative prognostic factor in the management of SSc-DUs.

References:

[1] Falanga V, Saap LJ, Ozonoff A. Wound bed score and its correlation with healing of chronic wound. Dermatol ther, 2006 Nov-Dec;19(69):383-90

Disclosure of Interests: None declared

DOI: 10.1136/annrheumdis-2020-eular.4234

\section{THU0340 DURATION AND SYSTEMIC SCLEROSIS SUBTYPE ARE ASSOCIATED WITH DIFFERENT GUT MICROBIOME PROFILES}

Y. Braun-Moscovici ${ }^{1,2}$, S. Ben Simon ${ }^{3}$, K. Dolnikov ${ }^{1,2}$, S. Giryes ${ }^{1}$, D. Markovits ${ }^{1,2}$, Y. Tavor ${ }^{1,2}$, K. Toledano ${ }^{1,2}$, A. Balbir-Gurman ${ }^{1,2}$, O. Koren ${ }^{3} .{ }^{1}$ Rambam Health Care Campus, B Shine Rheumatology Institute, Haifa, Israel; ${ }^{2}$ Rappaport Faculty of Medicine, Technion, Haifa, Israel; ${ }^{3}$ Faculty of Medicine, Bar Ilan University, Koren Lab, Safed, Israel

Background: A growing body of evidence suggests that the gut microbiota plays a significant role in the development of autoimmune diseases. Altered microbiota composition was associated with gastrointestinal and extraintestinal features in systemic sclerosis (SSc) patients.

Objectives: To look for differences in gut microbiota between SSc patients regarding disease duration, disease subset and occurrence of digital ulcers (DU). Methods: SSc patients seen at our center were recruited in a prospective study. The exclusion criteria included antibiotic or probiotic treatment during the month prior to recruitment, recent hospitalization, $\mathrm{BMI}>30$, diabetes mellitus or concomitant inflammatory bowel disease. Fecal samples were processed and 16S rRNA gene sequences were analyzed using the QIIME2 packageWeighted (quantitative) and unweighted (qualitative) UniFrac distances, alpha diversity for richness and homogeneity, taxa plots for species and phyla and ANCOM analyses were performed.

Results: During July 2018-May 2019, 26 SSc patients (mean age [SD] 53[12.7] years) and disease duration 8.8 [7.1] years) fulfilled the criteria and were willing to participate in the study. Thirteen patients had diffuse SSc, 16 patients had active DU, 8 patients had Raynaud's phenomenon only without DU, 2 patients had past DU. The microbiota was significantly more similar between patients without active DU compared to those with active DU $(P=0.024)$, but species richness did not differ. Patients with SSc duration less than 6 years had significantly different microbiota compared to long-lasting SSc (unweighted $\mathrm{PCoA}-\mathrm{q}=0.031$ ). Significant variations concerning quantitative and qualitative UniFrac distances $(q=0.063, q=0.005)$ and species richness $(q=0.009)$ were found among patients with diffuse compared to limited SSc. Limited SSc was

associated with greater species richness. Taxa plot analysis revealed higher relative abundance of Firmicutes in diffuse disease and of Actinobacteria and Bacteroidetes in limited SSc.

Conclusion: Disease duration, disease subset and active DU were associated with shifts in the microbiome of SSc patients. The impact of these changes on disease progression needs further elucidation.

Figure:

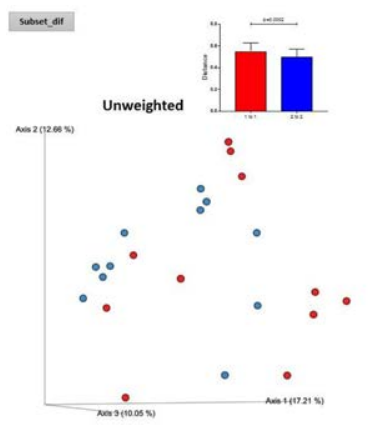

$\mathbf{q}=0.063$

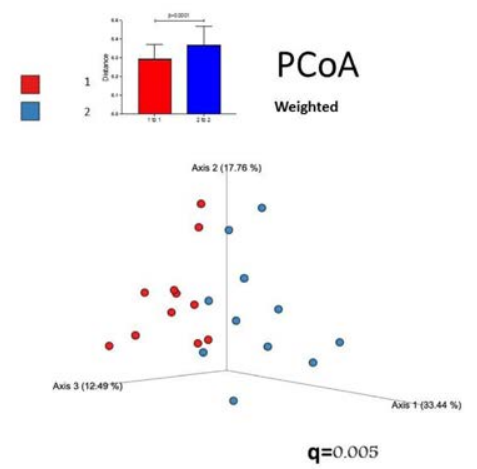

Disclosure of Interests: Yolanda Braun-Moscovici: None declared, Shira Ben Simon: None declared, Katya Dolnikov: None declared, Sami Giryes: None declared, Doron Markovits: None declared, Yonit Tavor: None declared, Kohava Toledano: None declared, Alexandra Balbir-Gurman Consultant of: Novartis, Omry Koren: None declared

DOI: 10.1136/annrheumdis-2020-eular.4865

\begin{tabular}{l|l}
\hline THU0341 & DIGITAL ARTERY VOLUME INDEX (DAVIX@) \\
PREDICTS THE ONSET OF FUTURE DIGITAL \\
ULCERS IN PATIENTS WITH SYSTEMIC \\
SCLEROSIS
\end{tabular}

K. Gjeloshi ${ }^{1}$, F. Danzo ${ }^{1}$, G. Lettieri ${ }^{2}$, G. Abignano ${ }^{2}$, M. Hinton ${ }^{3}$, A. M. Dean ${ }^{4,5}$, G. Cuomo', O. Kubassova ${ }^{3}$, F. Del Galdo ${ }^{* 4,5}$. ${ }^{1}$ University of Campania "Luigi Vanvitelli", Napoli, Italy; ${ }^{2}$ Istituto Reumatologico Lucano (IReL), Azienda Ospedaliera Regionale San Carlo, Potenza, Italy; ${ }^{3}$ IAG CEO, London, United Kingdom; ${ }^{4}$ NIHR Leeds Biomedical Research Centre, Leeds Teaching Hospitals NHS Trust, Leeds, United Kingdom; ${ }^{5}$ Leeds Institute of Rheumatic and Musculoskeletal Medicine, University of Leeds, Leeds, United Kingdom

Background: Neointima proliferation is a key pathologic feature of Systemic Sclerosis (SSc), causing arterial vessel narrowing and being the recognised culprit pathological lesion in Digital Ulcers (DUs), pulmonary artery hypertension and renal crisis. Nevertheless, there are no validated imaging techniques to assess the severity of vascular involvement in SSc. Digital Artery Volume index (DAVIX (C) is an MRI Time of flight angiography based quantitative score of digital arteries flow, without the need to administer contrast.

Objectives: To determine the value of DAVIX in predicting the onset of digital ulcers (DUs), the worsening of patient reported outcomes (PROs) and clinical parameters in SSc patients.

Methods: We enrolled 91 consecutive patients affected by Raynaud's phenomenon, 63 of which fulfilled the 2013 ACR/EULAR classification criteria for SSc and 28 had a score $<9$. The data collected included: pulmonary function tests (PFTs), nailfold capillaroscopy, modified Rodnan Skin Score (mRSS), and Scleroderma Health Assessment Questionnaire Disability Index (sHAQ-DI). DAVIX of the dominant hand was calculated as \% mean of the 4 fingers, employing IAG proprietary algorithm. The distribution was analysed with D'Agostino-Pearson normality test. Medians were compared by Mann-Whitney-Wilcoxon test, correlation with clinical parameters was performed using Spearman's or Pearson test, as appropriate (Prism 7).

Results: 78/91 patients were females and median disease duration was 4 years (IQR1.91-9). Complete historical and prospective follow-up data were available for 68 patients. DAVIX@ correlated with mRSS ( $r=-0.258, p=0.017)$, DLCO $\%$ $(r=0.338, p=0.008)$ and capillaroscopy pattern $(r=-0.388, p=0.001)$. In patients with DUs, DAVIX@ showed a stronger correlation with DLCO\% ( $r=0.786$, $p=0.048)$. DAVIX@ predicted the worsening of HAQ-DI $(r=-0.295, p=0.029)$, sHAQ $(r=-0.333, p=0.029)$ and VAS pain $(r=-0.269, p=0.038)$ independently of the presence of DUs. In the context of DU, 7 patients had DUs at baseline (5 with a positive history for DUs). 12 patients developed DUs within 12 months, 3 of them had DUs at baseline. 38 patients did not have either previous or current DUs, neither did they develop new DUs within 12 months. DAVIX of patients 